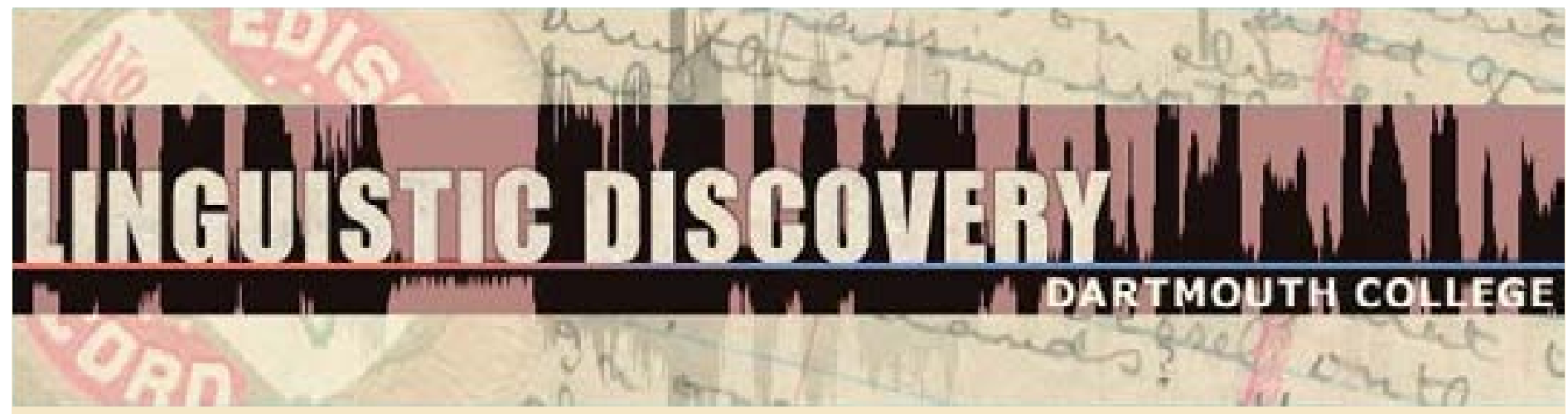

Volume 1

Issue 2 2002

\section{Archiving Electronic Journals}

Lenore A. Grenoble and Lindsay J. Whaley

Dartmouth College

doi: 10.1349/PS1.1537-0852.A.221

url: http://journals.dartmouth.edu/cgi-bin/WebObjects/ Journals.woa/1/xmlpage/1/article/221 


\title{
Archiving Electronic Journals
}

\author{
Lenore A. Grenoble and Lindsay J. Whaley \\ Dartmouth College
}

The choice to publish Linguistic Discovery in a purely electronic format has a range of benefits. The production costs are far lower than they would be for a print journal, which makes it possible to avoid subscription fees; the turn around time from receipt of a manuscript to publication can be cut dramatically in most cases; and most importantly, the inclusion of audio and video data, the potential for a dynamic presentation of information and the ability to link pages in a non-linear fashion open up intriguing possibilities about different ways of communicating discoveries about language. The electronic format of Linguistic Discovery also poses some challenges, two of which are of particular concern. First, there are technical issues about fonts, variations among browsers and platforms, search engines, and so on. Second, there are issues about archiving the journal. Although the former challenge is the one that our contributors tend to have the most questions about, and the one that adds most to the stress level of the editors, it is archiving that has the greater part in determining the continued success of the journal. For this reason, we felt it important to make some remarks on the topic.

In the electronic world, archiving must be considered from both a synchronic and diachronic perspective. Can an interested reader access the journal whenever she wants? Will an interested reader be able to access a current issue of the journal in a year, or five years, or fifty years from now? Such questions are more complicated than they might appear at first. Long term availability of information on the web entails long term maintenance of a site. How is this to be ensured? Web technology changes rapidly. How can we be confident that articles published in today's HTML and pdf files will be readable in the future? Journals need infusions of fresh ideas and energy from new editors from time to time to remain healthy. In the case of Linguistic Discovery, who maintains responsibility for the site when editorship changes? Does each editorship involve a switch in technical staff as well?

We have no definitive answers to such questions because the directions of evolving technology will dictate them in large measure. We can, however, offer provisional answers which will underscore our current thinking on the question of archiving.

While still in the process of creating Linguistic Discovery, we recognized the importance of getting a commitment from our home institution, Dartmouth College, to archive the materials. An institution needed to back the enterprise in order to ensure the journal's continuity, and it was convenient to have Dartmouth serve this role. From a more principled view, we see it as the prime mission of libraries to archive scholarly materials and make them accessible to society. This view, current in library communities today, takes the library to be a custodian of scholarly knowledge, regardless of format (as opposed to being a storehouse of only printed materials). With this principle in mind, Dartmouth's Baker-Berry Library now shoulders the responsibility of maintaining and archiving Linguistic Discovery.

Just how digital materials should be archived is a topic of great debate. What can be considered the more traditional approach is based on the assumption that having a single repository is the most secure means to ensure long-term maintenance and availability of materials. An antithetical idea is being investigated through a relatively new program, the LOCKSS initiative (LOCKSS="Lots of Copies Keeps Stuff Safe"), developed at Stanford University (http://lockss.stanford.edu/). LOCKSS has created software that can archive copies of 
material at multiple sites, based on the simple principle that the more copies there are, the greater the possibility that they will be available in the future. (LOCKSS sets the minimum number of storage sites at six, yet prefers 12). LOCKSS is still in an experimental stage, but has some very promising features. It is founded on the belief that libraries have a commitment to society to store, maintain, and make accessible scholarly knowledge.

An added benefit of LOCKSS for Linguistic Discovery is that it is designed to be inexpensive and easy to use. The cache machines used by LOCKSS to archive material are desktop PCs and have low system administration requirements. What this means in practical terms is that the equipment and personnel costs involved are relatively minor. Therefore, Dartmouth need not enter into potentially costly negotiations with other institutions in order to have Linguistic Discovery archived, and this in turn allows us to keep the journal free.

The LOCKSS model thus furnishes a provisional answer to how one will be able to access back issues of Linguistic Discovery into the indefinite future. The multiple storage sites should also mean that current issues of the journal should be available even if we are having server problems at Dartmouth, which happens more often than we would like to admit. It is likely that the first archiving site outside of Dartmouth for Linguistic Discovery will be established in the upcoming months; this will be a significant stage in the evolution of the journal.

What about the issue of how already published material will keep pace with changing technology? Of course, part of what it means to maintain the journal is a commitment to keeping past issues available and searchable on the Linguistic Discovery site. In addition, we burn CD's with copies of each issue; one set is stored in the Baker-Berry library, and in this form should be available indefinitely. Just as in the case of microfiche, film strips, and reel-to-reel tapes, we foresee libraries keeping equipment that allow access to digital information encoded in yesterday's technology. We find the non-virtual copies of the journal to be important just in case changes in information technologies make web-based technology obsolete.

This brings us to the final question. What happens with all this when the journal is administered somewhere other than Dartmouth? Several possibilities exist. The first is that a changing editorship will also entail a shift in institutional commitment to the journal, much the same as when a print journal shifts from one publishing house to another. In such a scenario Dartmouth's maintenance function would cease; its archiving role could or could not be continued, though given Dartmouth's participation in LOCKSS it is quite likely that it would. Another possibility is that Dartmouth would continue to have institutional oversight of the journal, and bear the responsibility for technical assistance and the like, despite the fact that the editor was employed elsewhere. At present, we see this as the more likely possibility, given the role of the Baker-Berry Library in the developing Linguistic Discovery, along with the commitment of the head librarian, Richard Lucier, to electronic publishing.

This all said, it is important to keep in mind that our thinking about archiving - and the technology available for it - are constantly evolving. We can only anticipate that electronic publications will be radically different in another decade. Given the current interest in electronic publishing, the technology for archiving scholarly materials will continue to change alongside changes in the technology for creating them. The LOCKSS program is still in the experimental stage. If successful, it will need constantly and persistently to evaluate its programs and develop new ones. At present, we see as critical the institutional commitment to be involved, in a very intimate way, with the publishing and archiving of Linguistic Discovery. 